\section{Assessment of the inter-platform reproducibility of ultrasound attenuation examination in nonalcoholic fatty liver disease}

\author{
Sun Kyung Jeon ${ }^{1}$, Jeong Min Lee ${ }^{1,2,3}$, ljin Joo ${ }^{1}$, Jeong Hee Yoon ${ }^{1}$ \\ ${ }^{1}$ Department of Radiology, Seoul National University Hospital, Seoul; ${ }^{2}$ Department of \\ Radiology, Seoul National University College of Medicine, Seoul; ${ }^{3}$ Institute of Radiation \\ Medicine, Seoul National University Medical Research Center, Seoul, Korea
}

Purpose: This study aimed to assess the inter-platform reproducibility of ultrasound attenuation examination in patients with nonalcoholic fatty liver disease (NAFLD).

Methods: Between March 2021 and April 2021, patients with clinically suspected or known NAFLD were prospectively enrolled; each patient underwent ultrasound attenuation examinations with three different platforms (Attenuation Imaging [ATI], Canon Medical System; Tissue Attenuation Imaging [TAI], Samsung Medison; and Ultrasound-Guided Attenuation Parameter [UGAP], GE Healthcare) on the same day. The mean attenuation coefficient (AC) values of the three platforms were compared using repeated-measures analysis of variance with the Bonferroni correction. To evaluate inter-platform reproducibility, the $A C$ values obtained for each platform were compared using Bland-Altman analysis with the calculation of $95 \%$ limits of agreement (LOA), intraclass correlation coefficients (ICCS), and coefficients of variation (CVs).

Results: Forty-six patients (23 men; mean age \pm standard deviation, 52.3 \pm 12.4 years) were enrolled. The mean $A C$ values showed significant differences among the three platforms $(0.75 \pm 0.12,0.80 \pm 0.11$, and $0.74 \pm 0.09 \mathrm{~dB} / \mathrm{cm} / \mathrm{MHz}$ for ATI, TAI, and UGAP, respectively; $\mathrm{P}<0.001)$. For inter-platform reproducibility, the $95 \% \mathrm{LOAs}$ were -0.22 to $0.11 \mathrm{~dB} / \mathrm{cm} / \mathrm{MHz}$ between ATI and TAI, -0.17 to $0.18 \mathrm{~dB} / \mathrm{cm} / \mathrm{MHz}$ between ATI and UGAP, and -0.08 to $0.20 \mathrm{~dB} /$ $\mathrm{cm} / \mathrm{MHz}$ between TAI and UGAP, respectively. The pairwise ICCs were $0.790-0.797$ in terms of absolute agreement among the three platforms; the CVs were $8.23 \%-9.47 \%$.

Conclusion: The AC values obtained from different ultrasound attenuation examination platforms showed significant differences, with significant inter-platform variability. Therefore, the AC values measured using different ultrasound attenuation examination techniques should not be used interchangeably for longitudinal follow-up of patients with NAFLD.

Keywords: Ultrasonography; Liver; Fatty liver; Nonalcoholic fatty liver disease

Key points: Attenuation coefficient values obtained from different ultrasound attenuation examination platforms showed significant differences, with substantial inter-platform variability.

e-ultrasonography.org

Ultrasonography 41(2), April 2022

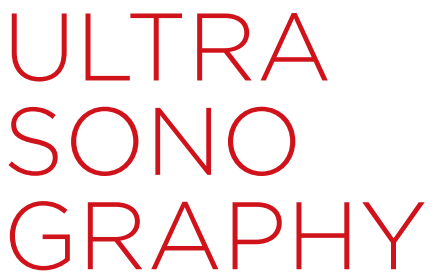

ORIGINAL ARTICLE

https://doi.org/10.14366/usg.21167 pISSN: 2288-5919 • elSSN: 2288-5943 Ultrasonography 2022;41:355-364

Received: August 9, 2021

Revised: October 7, 2021

Accepted: October 31, 2021

Correspondence to:

Jeong Min Lee, MD, Department of Radiology, Seoul National University Hospital and Seoul National University College of Medicine, 101 Daehak-ro, Jongno-gu, Seoul 03080, Korea

Tel. +82-2-2072-2057

Fax. +82-2-743-6385

E-mail: jmsh@snu.ac.kr

This is an Open Access article distributed under the terms of the Creative Commons Attribution NonCommercial License (http://creativecommons.org/ licenses/by-nc/4.0/) which permits unrestricted noncommercial use, distribution, and reproduction in any medium, provided the original work is properly cited.

Copyright (C) 2022 Korean Society of Ultrasound in Medicine (KSUM)

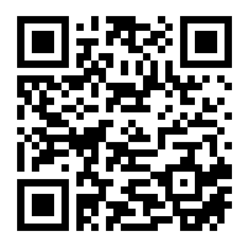

How to cite this article:

Jeon SK, Lee JM, Joo I, Yoon JH. Assessment of the inter-platform reproducibility of ultrasound attenuation examination in nonalcoholic fatty liver disease. Ultrasonography. 2022 Apr;41(2):355-364. 


\section{Introduction}

The prevalence of nonalcoholic fatty liver disease (NAFLD) is increasing globally, and NAFLD is emerging as the most common type of chronic liver disease in many parts of the world [1]. NAFLD comprises a spectrum of conditions ranging from simple steatosis to nonalcoholic steatohepatitis (NASH), which can progress to cirrhosis $[2,3]$. The progression of NAFLD to NASH has been known to be stopped or reversed by the early detection and treatment of hepatic steatosis [4]. Although liver biopsy remains the current gold standard for the diagnosis of hepatic steatosis, the invasiveness of biopsy methods and occurrence of sampling errors underscores the need for noninvasive diagnostic tools [5]. Several imaging modalities have been used for the noninvasive assessment of hepatic steatosis [6-8]. Magnetic resonance spectroscopy and chemical shiftencoded magnetic resonance imaging-based proton density fat fraction (MRI-PDFF) are used as validated reference standards with excellent accuracy and reproducibility for the evaluation of hepatic steatosis $[6,7]$. However, MRI-based techniques may not be costeffective or easily accessible for the clinical screening of NAFLD considering the high prevalence of this disease [9]. Although B-mode ultrasonography is a commonly used test for hepatic steatosis and is advantageous in terms of its wide availability, the results of this test are subjective, operator-dependent, and not quantifiable $[8,10]$.

In recent years, various quantitative ultrasound techniques, including the speed of sound, ultrasound attenuation, and backscatter coefficient, have been developed for the quantification of hepatic fat [11-14]. Among various quantitative ultrasound techniques, several ultrasound manufacturers have developed software for quantifying the attenuation of ultrasound beams, which increases as hepatic steatosis progresses $[15,16]$. The controlled attenuation parameter, which is measured by transient elastography, can provide objective measurements of hepatic steatosis; however, it cannot provide B-mode ultrasound images and is known to be affected by the patients' age, skin-to-liver capsule distance, and body mass index (BMI) $[17,18]$. Several ultrasound manufacturers have recently developed B-mode ultrasound-guided attenuation examination techniques. Compared to conventional ultrasonography, ultrasound-guided attenuation examination is less operatordependent and still has the inherent advantages of ultrasonography, such as wide availability, real-time capability, and relatively low cost [19]. In previous studies, ultrasound attenuation examination techniques have demonstrated good accuracy in the diagnosis and grading of hepatic steatosis, with excellent inter-examiner or intraexaminer reliability [20-23].

However, little is known about the reproducibility of ultrasound attenuation coefficient $(A C)$ values measured using multiple platforms from different scanner vendors. High inter-platform reproducibility of ultrasound attenuation examination is essential for its clinical application in patients with NAFLD for purposes including the diagnosis, monitoring, and follow-up of hepatic steatosis. Therefore, this study aimed to assess the inter-platform reproducibility of ultrasound attenuation examination in patients with NAFLD.

\section{Materials and Methods}

\section{Compliance with Ethical Standards}

This single-center, prospective study was approved by the institutional review board of Seoul Nationl University Hospital (IRB No. 2102-044-1195), and written informed consent was obtained from all participants.

\section{Study Population}

Patients who met the eligibility criteria between March 2021 and April 2021 and provided written informed consent were prospectively enrolled in this study. The inclusion criteria were as follows: (1) age $\geq 18$ years and (2) referral to the radiology department for ultrasonographic evaluation of the liver because of suspected or known NAFLD. The exclusion criteria were as follows: (1) age less than 18 years; the (2) presence of clinical, laboratory, or histological evidence of liver disease other than NAFLD; (3) excessive alcohol consumption ( $\geq 14$ and $\geq 7$ drinks per week for men and women, respectively); (4) steatogenic or hepatotoxic medication use; and (5) history of liver surgery.

\section{B-Mode Ultrasound and Ultrasound Attenuation Examinations}

For each patient, conventional B-mode ultrasound and ultrasound attenuation examinations were performed by one of two body radiologists (S.K.J. and J.M.L., each with more than 7 years of experience in abdominal ultrasound examinations). All patients were requested to fast for more than 4 hours prior to the ultrasound examination.

\section{$B$-mode ultrasound examinations}

First, a conventional B-mode ultrasound examination was performed using an ultrasound system (Aplio i900, Canon Medical Systems, Tochigi, Japan) with a 1-8 MHz convex probe. The visual score of hepatic steatosis was recorded by the operator as follows: 0 , no steatosis; 1, mild steatosis; 2, moderate steatosis; and 3, severe steatosis. These scores were based on Hamaguchi's scoring system using the following ultrasound imaging features: bright liver, increased hepatorenal echo contrast, deep attenuation, and vessel blurring [24]. Additionally, the skin-to-liver capsule distance (mm) 
was measured.

\section{Ultrasound attenuation examinations}

For each patient, after the B-mode ultrasound examination, two sessions of ultrasound attenuation examinations were performed by the same radiologist on the same day. Each session was performed using three different ultrasound attenuation examination platforms: Attenuation Imaging (ATI) using Aplio i900 (Canon Medical System), Tissue Attenuation Imaging (TAI) using RS 85 (Samsung Medison, Seoul, Korea), and Ultrasound-Guided Attenuation Parameter (UGAP) using LOGIQ E10 (GE Healthcare, Chicago, IL, USA). The ultrasound attenuation examinations were performed for the right lobe of the liver through an intercostal plane near the level of the hepatic hilum during breath-holding. The radiologists tried to perform the examinations at the same liver location in each patient. In addition, although there were no specific instructions on the timing of patients' breathing, the examination was performed with a constant timing of breathing and at the point where the same view was seen to the extent possible in each patient.

In the ATI examination, with activation of the ATI mode, a fanshaped color-coded sampling box was positioned in the hepatic parenchyma at least $2 \mathrm{~cm}$ below the liver capsule while avoiding areas with large vessels, focal fat sparing or deposition, and reverberation artifacts or shadowing. Structures other than the hepatic parenchyma, such as vascular structures, were automatically excluded from the sampling box. Thereafter, a $2 \times 4 \mathrm{~cm}$ fan-shaped region of interest (ROI) was placed within the sampling box, and the $A C$ value $(\mathrm{dB} / \mathrm{cm} / \mathrm{MHz})$ was calculated and displayed (Fig. 1A).

For the TAl examination, with the selection of a function key for TAl, a $2 \times 3 \mathrm{~cm}$ fan-shaped ROI with a color-coded map was generated (Fig. 1B). The ROI box was placed in a right lobe of the liver at least $2 \mathrm{~cm}$ below the liver capsule while avoiding areas with large vessels, focal fat sparing or deposition, and reverberation artifacts or shadowing. Areas with significant errors in the calculation of parameters, such as vascular structures, were automatically excluded from the maps. The $A C$ value $(\mathrm{dB} / \mathrm{cm} / \mathrm{MHz})$ was automatically calculated and provided.

For the UGAP examination, with activation of the UGAP mode, a color-coded map was generated where the signal quality was sufficiently high to perform a measurement (quality map). An $\mathrm{ROI}$ with a length of $65 \mathrm{~mm}$ was placed within the color-coded map while avoiding areas with large vessels, focal fat sparing or deposition, and reverberation artifacts or shadowing, and the AC value was automatically calculated (Fig. 1C).

For both ATI and TAI examinations, the reliability of measurement was described as an $R^{2}$ value, and the operator attempted to obtain $A C$ values with an $R^{2}$ value $\geq 0.6$. For the UGAP examination, an unreliable area was presented as a vacancy on the quality map, and the operator attempted to obtain $A C$ values by avoiding that area. Five consecutive measurements were performed for each examination, and the mean values of the five measurements were used for analysis.

\section{Statistical Analysis}

Continuous values were summarized as means with standard deviations, and categorical variables were summarized as counts with percentages. Pearson correlation coefficients were calculated between the AC values of ATI and TAI, ATI and UGAP, and TAI and UGAP. Repeated-measures one-way analysis of variance (ANOVA) with the Bonferroni post-hoc test was performed to compare the mean $A C$ values of different ultrasound attenuation examination platforms. In addition, scatter plots and Bland-Altman plots were generated for the AC values of the different platforms.

To evaluate the inter-platform reproducibility of the different ultrasound attenuation examination platforms and inter-session reproducibility of each platform, a Bland-Altman analysis with 95\% limits of agreement (LOAs) was employed for the mean AC values of the different platforms. Intraclass correlation coefficients (ICCS) with $95 \%$ confidence intervals (Cls) and coefficients of variation (CVs, \%) were calculated [25]. ICC values were calculated based on a single-unit two-way mixed-effects ANOVA model in which the patient was treated as a random effect and the platform was treated as a fixed effect. The ICC for absolute agreement was also reported. Agreement using ICCs was classified using the following criteria: $\geq 0.90$, excellent; $\geq 0.75$ to $<0.90$, good; $\geq 0.50$ to $<0.75$, moderate; and $<0.50$, poor [26]. Pearson correlation coefficients were calculated to evaluate the correlations between ultrasound attenuation examination platforms and were categorized using the following criteria: $0-0.19$, very weak; $0.2-0.39$, weak; $0.40-0.59$, moderate; $0.60-0.79$, strong; and $0.80-1.0$, very strong [27]. In addition, to evaluate the inter-platform reproducibility of different ultrasound attenuation examination platforms according to the visual grade of hepatic steatosis, the Bland-Altman 95\% LOAs, ICCS, CVs, and Pearson correlation coefficients were calculated in patients with no or mild steatosis and moderate to severe steatosis (Supplementary Table 1). To evaluate whether inter-platform variability is affected by the patient's BMI and skin-to-liver capsule distance, Pearson correlation coefficients were calculated between the absolute between-platform differences in $\mathrm{AC}$ values and $\mathrm{BMI}$; and between the absolute inter-platform differences in $A C$ values and the skin-to-liver capsule distance. All statistical analyses were performed using commercially available software (SPSS version 25, IBM Corp., Armonk, NY, USA; MedCalc version 18, MedCalc Software, Mariakerke, Belgium), with P-values $<0.05$ considered to 


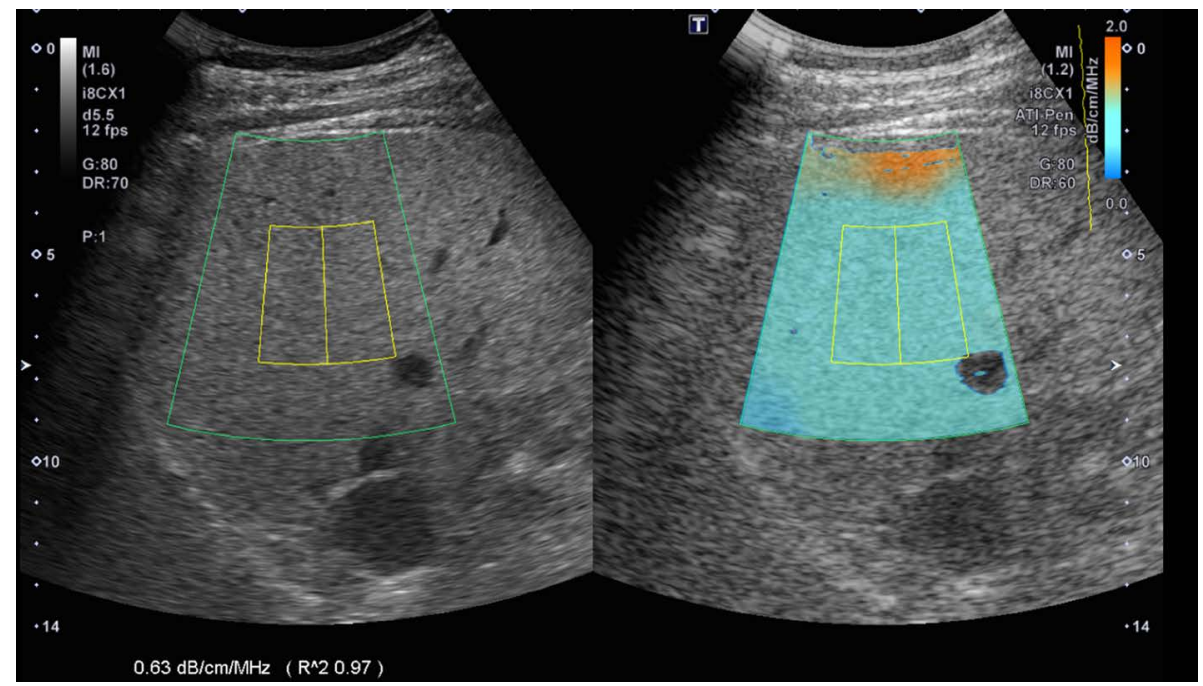

A

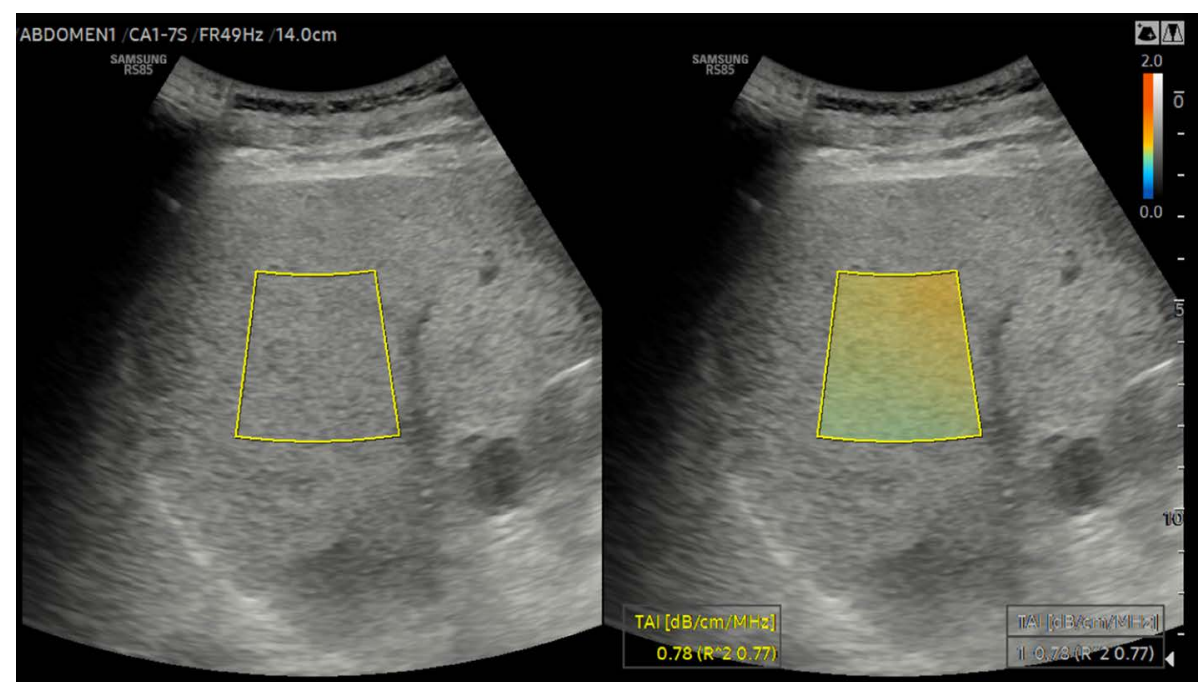

B

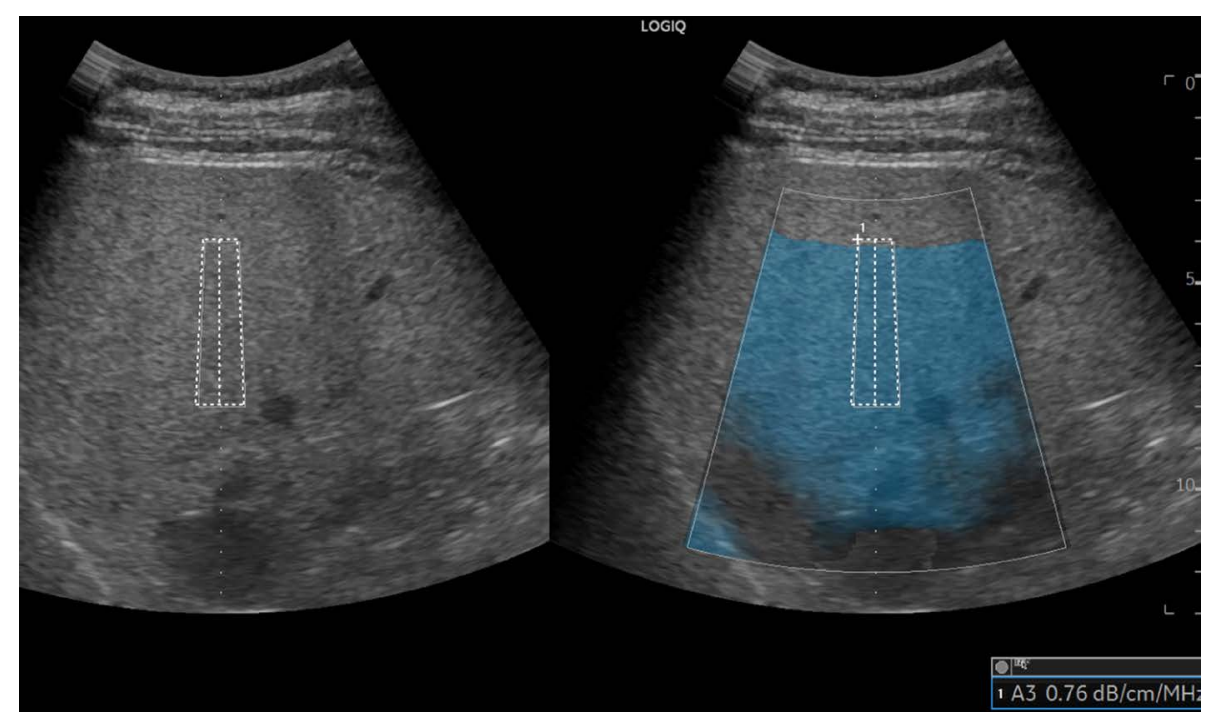

C
Fig. 1. Measurement of attenuation coefficients $(\mathrm{AC})$ using three ultrasound attenuation examination platforms.

$A C$ values were measured in each patient using Attenuation Imaging (Canon Medical System) (A), Tissue Attenuation Imaging (Samsung Medison) (B), and UltrasoundGuided Attenuation Parameter (GE Healthcare) (C), respectively. 
indicate a statistically significant difference.

\section{Results}

Forty-six patients ( 23 men; mean age, $52.3 \pm 12.4$ years; range, 24 to 80 years) were enrolled in the study and their data included in the analyses. The demographic characteristics of the study cohort are summarized in Table 1. The patients had an average BMI of $26.2 \pm 3.0 \mathrm{~kg} / \mathrm{m}^{2}$ and an average skin-to-liver capsule distance of $19.9 \pm 4.1 \mathrm{~mm}$. On visual assessment, patients had either no $(n=7)$, mild $(n=14)$, moderate $(n=17)$, or severe hepatic steatosis $(n=8)$.

Table 1. Patient characteristics

\begin{tabular}{lc}
\hline \multicolumn{1}{c}{ Variable } & Value $(\mathrm{n}=46)$ \\
\hline Age (year) & $52.3 \pm 12.4(24-80)$ \\
Sex & $23(50.0)$ \\
Male & $23(50.0)$ \\
Female & $26.2 \pm 3.0(19.0-34.6)$ \\
BMI (kg/m $\left.{ }^{2}\right)$ & $19.9 \pm 4.1(11-32)$ \\
Skin-to-liver capsule distance (mm) & $31.2 \pm 17.1(14-80)$ \\
Aspartate aminotransferase (IU/L) & $42.1 \pm 31.4(8-145)$ \\
Alanine aminotransferase (IU/L) & $7(15.2)$ \\
Visual hepatic steatosis grade & $14(30.4)$ \\
No steatosis & $17(37.0)$ \\
Mild steatosis & $8(17.4)$ \\
Moderate steatosis & \\
Severe steatosis & \\
AC measured using ultrasound attenuation & \\
examination & \\
ATI & $0.75 \pm 0.12(0.50-0.98)$ \\
TAl & $0.80 \pm 0.11(0.61-1.08)$ \\
UGAP & $0.74 \pm 0.09(0.52-0.93)$ \\
\hline Va &
\end{tabular}

Values are presented as mean \pm standard deviation (range) or number (\%).

BMI, body mass index; AC, attenuation coefficient; ATI, Attenuation Imaging (Canon Medical System); TAl, Tissue Attenuation Imaging (Samsung Medicine); UGAP, Ultrasound-Guided Attenuation Parameter (GE Healthcare).

\section{Inter-platform Reproducibility of AC Values between ATI, TAI, and UGAP}

The mean AC values were $0.75 \pm 0.12$ for ATI, $0.80 \pm 0.11$ for TAl, and $0.74 \pm 0.09 \mathrm{~dB} / \mathrm{cm} / \mathrm{MHz}$ for UGAP, and these values were strongly correlated with each other (ATI and TAI, $r=0.73 ; 95 \% \mathrm{Cl}$, 0.56 to $0.84 ; \mathrm{P}<0.001 ; \mathrm{ATI}$ and $\mathrm{UGAP}, \mathrm{r}=0.68 ; 95 \% \mathrm{Cl}, 0.48$ to $0.81 ; \mathrm{P}<0.001$; and TAl and UGAP, $\mathrm{r}=0.78 ; 95 \% \mathrm{Cl}, 0.63$ to 0.87 ; $P<0.001)$. The mean $A C$ value for TAl was significantly higher than that for ATI and UGAP ( $P<0.001$ for both), whereas there was no significant difference between the mean $A C$ values for ATI and UGAP (P>0.99) (Table 2, Fig. 2).

The Bland-Altman analysis showed a bias across $A C$ values for different ultrasound attenuation examination platforms, with a mean difference of $-0.05 \mathrm{~dB} / \mathrm{cm} / \mathrm{MHz}$ between ATI and TAl, $0.01 \mathrm{~dB} / \mathrm{cm} /$ $\mathrm{MHz}$ between ATI and UGAP, and $0.06 \mathrm{~dB} / \mathrm{cm} / \mathrm{MHz}$ between TAl and UGAP. The $95 \%$ LOAs of the mean AC values ranged from -0.22 to $0.11 \mathrm{~dB} / \mathrm{cm} / \mathrm{MHz}$ for ATI and TAl, from -0.17 to $0.18 \mathrm{~dB} / \mathrm{cm} / \mathrm{MHz}$ for

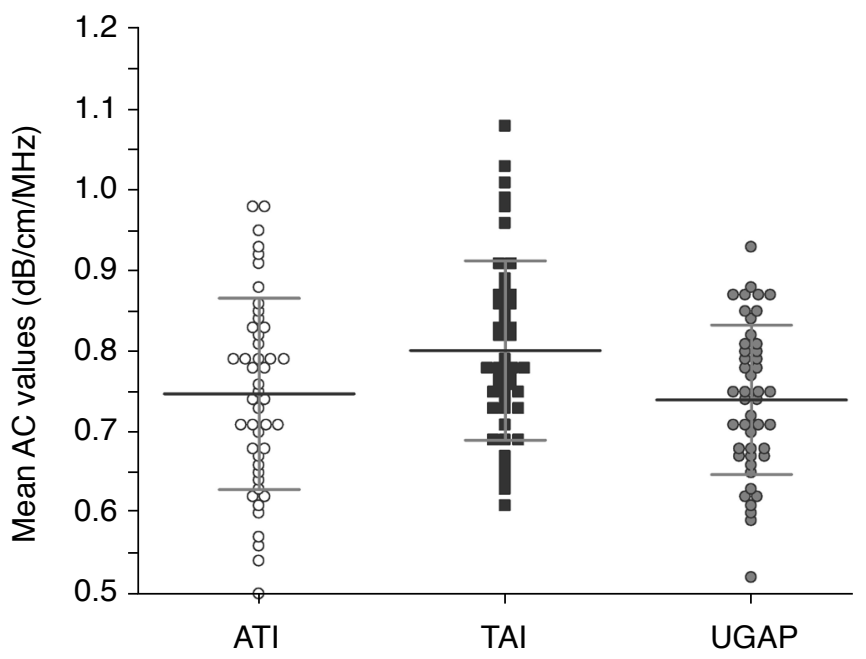

Fig. 2. Distribution of mean attenuation coefficients obtained using three ultrasound attenuation examination platforms. AC, attenuation coefficient; ATI, Attenuation Imaging; TAI, Tissue Attenuation Imaging; UGAP, Ultrasound-Guided Attenuation Parameter.

Table 2. Mean AC values obtained using different ultrasound attenuation examination platforms

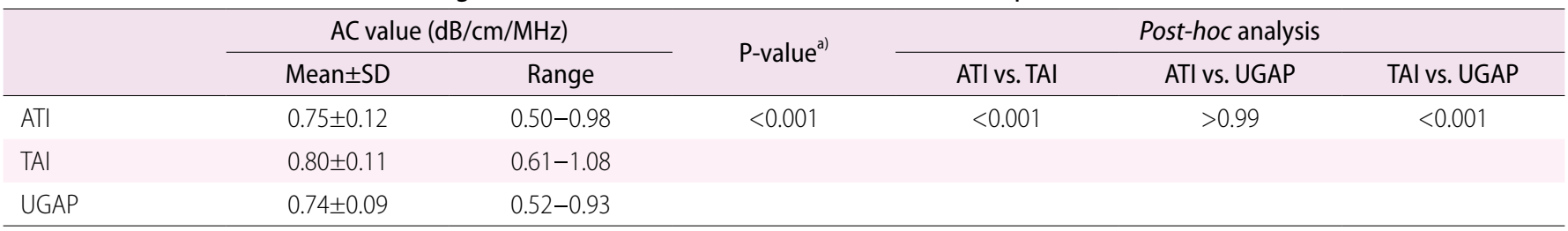

AC, attenuation coefficient; SD, standard deviation; ATI, Attenuation Imaging (Canon Medical System); TAl, Tissue Attenuation Imaging (Samsung Medicine); UGAP, UltrasoundGuided Attenuation Parameter (GE Healthcare).

a)P-values were calculated using repeated-measures analysis of variance with Bonferroni post-hoc analysis. 


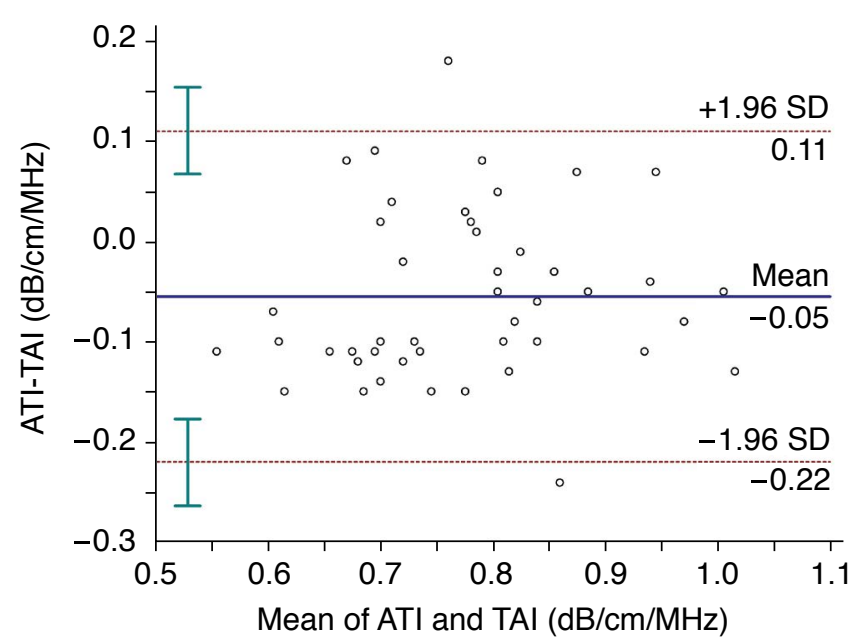

A

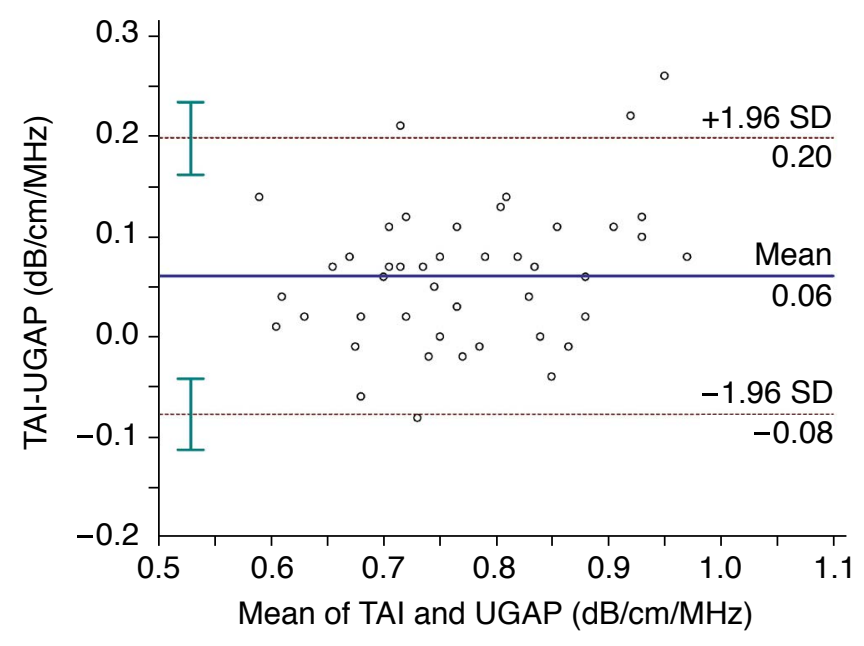

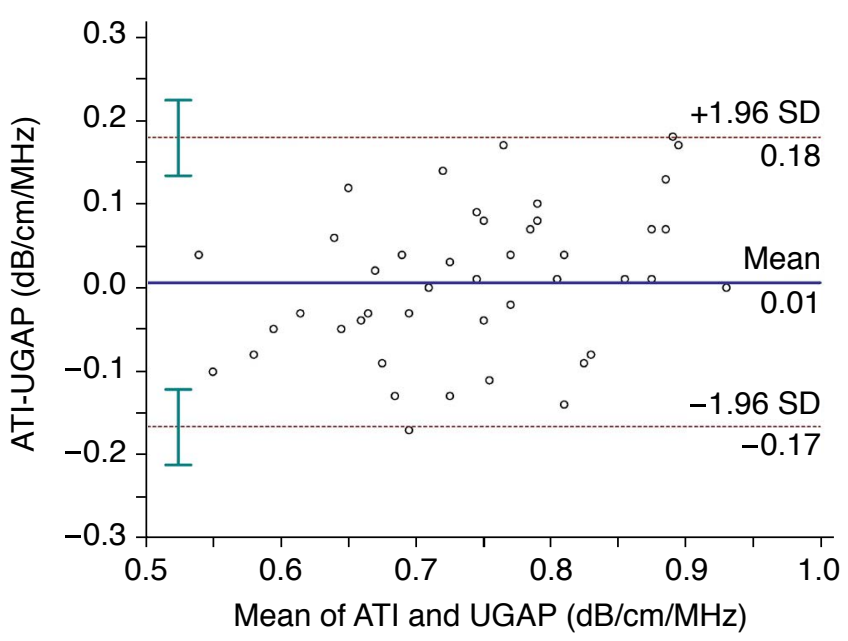

B

Fig. 3. Bland-Altman plots of the ultrasound attenuation examination platforms.

The Bland-Altman plots demonstrate differences in attenuation coefficient (AC) values between Attenuation Imaging (ATI) and Tissue Attenuation Imaging (TAI) (A), ATI and Ultrasound-Guided Attenuation Parameter (UGAP) (B), and TAI and UGAP (C). The solid blue line in the middle represents the mean $A C$ values obtained from each pair of the three platforms, and the dotted brown lines indicate \pm 1.96 standard deviations (SDs), with the associated $95 \%$ confidence intervals indicated by the green bars.

C

Table 3. Inter-platform reproducibility of $A C$ values between three ultrasound attenuation examination platforms

\begin{tabular}{lccccc}
\hline & Mean bias (dB/cm/MHz) & BALA (dB/cm/MHz) & ICC & CV (\%) & Pearson r \\
\hline ATI vs. TAI & $-0.05(-0.08$ to -0.03$)$ & -0.22 to 0.11 & $0.797(0.495$ to 0.904$)$ & $9.47(7.91$ to 10.80$)$ & 0.733 (0.562 to 0.844$)$ \\
ATI vs. UGAP & $0.01(-0.02$ to 0.03$)$ & -0.17 to 0.18 & $0.794(0.628$ to 0.886$)$ & 8.27 (6.79 to 9.54) & 0.675 (0.479 to 0.807$)$ \\
TAI vs. UGAP & $0.06(0.04$ to 0.08$)$ & -0.08 to 0.20 & $0.790(0.273$ to 0.915$)$ & 8.23 (6.26 to 9.82) & 0.778 (0.631 to 0.872) \\
\hline
\end{tabular}

Numbers in parentheses are $95 \%$ confidence intervals.

AC, attenuation coefficient; BALA, Bland-Altman 95\% limits of agreement; ICC, intraclass correlation coefficient; CV, coefficient of variation; ATI, Attenuation Imaging; TAI,

Tissue Attenuation Imaging; UGAP, Ultrasound-Guided Attenuation Parameter.

ATI and UGAP, and from -0.08 to $0.20 \mathrm{~dB} / \mathrm{cm} / \mathrm{MHz}$ for TAl and UGAP (Table 3, Fig. 3).

The pairwise ICCS of AC values for different ultrasound attenuation examination platforms ranged from 0.790 to 0.797 (0.797 for ATI and TAl; 0.794 for ATI and UGAP; and 0.790 for TAI and UGAP), indicating good agreement. The CVs were $9.47 \%$ for ATI and TAI, $8.27 \%$ for ATI and UGAP, and $8.23 \%$ for TAI and UGAP (Table 3).
Inter-platform Reproducibility and Potential Confounding Factors

BMI was not correlated with the absolute inter-platform difference in AC values: ATI and TAI, $r=0.008, P=0.957 ; A T I$ and UGAP, $r=0.031$, $P=0.839 ;$ TAl and UGAP, $r=0.077, P=0.077$. Additionally, the skinto-liver capsule distance was not related with the absolute interplatform difference in AC values: ATI and TAI, $r=0.091, P=0.548$; 
Table 4. Inter-session pairwise repeatability of AC values obtained using different ultrasound attenuation examination platforms

\begin{tabular}{|c|c|c|c|c|c|c|c|c|}
\hline & \multicolumn{2}{|c|}{ AC value $(\mathrm{dB} / \mathrm{cm} / \mathrm{MHz})$} & \multirow{2}{*}{ P-value ${ }^{a)}$} & \multirow{2}{*}{ Mean bias } & \multirow{2}{*}{ BALA } & \multirow{2}{*}{ ICC } & \multirow{2}{*}{ CV (\%) } & \multirow{2}{*}{ Pearson $r$} \\
\hline & 1st session & 2nd session & & & & & & \\
\hline ATI & $0.75 \pm 0.12$ & $0.74 \pm 0.12$ & 0.085 & 0.00 & -0.03 to 0.03 & 0.962 (0.931 to 0.979$)$ & $4.9(0.9$ to 7.0$)$ & 0.931 (0.878 to 0.961$)$ \\
\hline TAl & $0.80 \pm 0.11$ & $0.81 \pm 0.11$ & 0.126 & -0.01 & -0.10 to 0.08 & 0.957 (0.922 to 0.976$)$ & 3.9 (1.2 to 5.3$)$ & 0.920 (0.858 to 0.955$)$ \\
\hline UGAP & $0.74 \pm 0.09$ & $0.75 \pm 0.09$ & 0.385 & 0.00 & -0.07 to 0.06 & 0.962 (0.931 to 0.979$)$ & 3.4 (2.6 to 4.0$)$ & 0.926 (0.869 to 0.959$)$ \\
\hline
\end{tabular}

Numbers in parentheses are $95 \%$ confidence intervals.

AC, attenuation coefficient; BALA, Bland-Altman limits of agreement; ICC, intraclass correlation coefficient; CV, coefficient of variation; ATI, Attenuation Imaging; TAl, Tissue Attenuation Imaging; UGAP, Ultrasound-Guided Attenuation Parameter.

a) P-values were calculated using the paired Student t-test (two-tailed).

ATI and UGAP, $r=-0.054, P=0.721$; and TAI and UGAP, $r=-0.043$, $P=0.778$. The inter-platform reproducibility improved with an increasing number of acquisitions, averaged from one to five. When five acquisitions were used, the inter-platform ICCS for AC were $0.79-0.80$ in terms of the absolute agreement, and the CVs were $8.23 \%-9.47 \%$ (Supplementary Table 2).

\section{Inter-session Reproducibility of Each Ultrasound Attenuation Examination Platform}

The inter-session reproducibility of each ultrasound attenuation examination platform is summarized in Table 4. For each platform, the overall inter-session reproducibility was excellent, with ICCS of 0.962 ( $95 \% \mathrm{Cl}, 0.931$ to 0.979 ) for ATI, 0.957 (95\% Cl, 0.922 to $0.976)$ for TAl, and 0.962 ( $95 \% \mathrm{Cl}, 0.931$ to 0.979$)$ for UGAP. The CVs were $4.9 \%(95 \% \mathrm{Cl}, 0.9$ to 7.0$)$ for ATI, 3.9\% $(95 \% \mathrm{Cl}, 1.2$ to 5.3) for TAl, and $3.4 \%(95 \% \mathrm{Cl}, 2.6$ to 4.0$)$ for UGAP.

The Bland-Altman analysis showed a slight bias across AC values between the two sessions of each ultrasound attenuation examination platform, with a mean difference of $0 \mathrm{~dB} / \mathrm{cm} / \mathrm{MHz}$ for ATI and UGAP and $-0.01 \mathrm{~dB} / \mathrm{cm} / \mathrm{MHz}$ for TAI. The $95 \%$ LOAs of the mean $A C$ values ranged from -0.03 to $0.03 \mathrm{~dB} / \mathrm{cm} / \mathrm{MHz}$ for ATI, from -0.10 to $0.08 \mathrm{~dB} / \mathrm{cm} / \mathrm{MHz}$ for TAl, and from -0.07 to $0.06 \mathrm{~dB} /$ $\mathrm{cm} / \mathrm{MHz}$ for UGAP.

\section{Discussion}

In clinical practice, it is a highly likely that several different ultrasound systems are used for the follow-up of patients with NAFLD. Therefore, excellent inter-platform reproducibility is an essential aspect of the wide clinical use of ultrasound attenuation examination for longitudinal follow-up in the monitoring of treatment response. In this study, although each ultrasound attenuation examination platform had excellent inter-session reproducibility, significant inter-platform variability was observed in the mean $A C$ values measured using the different platforms. Thus, $A C$ values measured using different ultrasound attenuation examination techniques should not be used interchangeably for longitudinal follow-up, and different cutoff values for steatosis grading should be applied to different ultrasound attenuation examination platforms.

Only a few studies have investigated the inter-platform reproducibility of ultrasound attenuation examination. Previous studies by Han et al. $[28,29]$ assessed the inter-platform reproducibility of $A C$ values using ultrasound radiofrequency data obtained from two different clinical ultrasound systems and reported an ICC of 0.77 , a Pearson correlation coefficient of 0.81 , and a small standard deviation of measurements $(<0.07 \mathrm{~dB} / \mathrm{cm} / \mathrm{MHz})$; therefore, the authors concluded that the inter-platform reproducibility of $A C$ values was good. However, in those studies, although ultrasound radiofrequency data were obtained using different ultrasound platforms, all $A C$ values were obtained in common using an offline software program in MATLAB that used the same method of calculating $A C$ values. However, this workflow is quite different from routine clinical practice. In routine clinical practice, each clinical ultrasound machine uses a different system and software methods of calculating AC values. Instead, the present study used three clinical ultrasound machines, each with its own ultrasound attenuation examination platform, which may more closely reflect the real clinical application of ultrasound attenuation examination. In the present study, significant inter-platform variability was observed, which was not affected by BMI or skin-to-liver capsule distance. As this inter-platform variability could be attributed to several systemrelated factors and the software's method of calculating ACs, it could be consistently observed across the different ultrasound attenuation examination systems.

In the present study, the pairwise ICCs of AC values from different ultrasound attenuation examination platforms were 0.790-0.797, indicating good agreement, which corresponds to the findings of a previous study by Han et al. [28]. However, the $95 \%$ LOAs of the absolute difference in mean $A C$ values were quite large and were thought to be clinically unacceptable $(-0.22$ to $0.11 \mathrm{~dB} / \mathrm{cm} /$ $\mathrm{MHz}$ for ATI and TAl, -0.17 to $0.18 \mathrm{~dB} / \mathrm{cm} / \mathrm{MHz}$ for ATI and UGAP, and -0.08 to $0.20 \mathrm{~dB} / \mathrm{cm} / \mathrm{MHz}$ for TAl and UGAP). Many previous 
studies have reported high diagnostic performance of ultrasound attenuation examination for diagnosing hepatic steatosis and grading severity, but there is wide variation in the optimal cutoff values for diagnosing hepatic steatosis reported in each study, ranging from 0.59 to $0.69 \mathrm{~dB} / \mathrm{cm} / \mathrm{MHz}$ for ATI (0.59 to $0.69 \mathrm{~dB} /$ $\mathrm{cm} / \mathrm{MHz}$ with MRI-PDFF as the reference standard; 0.64 to 0.69 $\mathrm{dB} / \mathrm{cm} / \mathrm{MHz}$ with histopathology as the reference standard); 0.88 $\mathrm{dB} / \mathrm{cm} / \mathrm{MHz}$ for TAI (with MRI-PDFF as the reference standard), and from 0.53 to $0.60 \mathrm{~dB} / \mathrm{cm} / \mathrm{MHz}$ for UGAP $(0.53 \mathrm{~dB} / \mathrm{cm} / \mathrm{MHz}$ with histopathology as the reference standard; $0.60 \mathrm{~dB} / \mathrm{cm} / \mathrm{MHz}$ with MRI-PDFF as the reference standard [20-22,30]. Moreover, the optimal cutoff values for discriminating each grade of hepatic steatosis (mild [S1], moderate [S2], and severe [S3]) showed minimal gaps (range, 0.02 to $0.10 \mathrm{~dB} / \mathrm{cm} / \mathrm{MHz}$ ) [20-22,30]. Considering the minimal gradual change in $A C$ values according to the grade of hepatic steatosis, the calculated $95 \%$ LOAs of the absolute difference in $A C$ values in the present study were thought to be too large to be clinically acceptable.

Our study results demonstrated excellent inter-session reproducibility of each ultrasound attenuation examination platform. Of note, the mean bias and $95 \%$ LOAs were quite minimal (95\% LOAs of mean AC values, -0.03 to $0.03 \mathrm{~dB} / \mathrm{cm} / \mathrm{MHz}$ for $A T I,-0.10$ to $0.08 \mathrm{~dB} / \mathrm{cm} /$ $\mathrm{MHz}$ for TAI, and -0.07 to $0.06 \mathrm{~dB} / \mathrm{cm} / \mathrm{MHz}$ for UGAP, respectively). These results are in line with previous studies that reported excellent intra-examiner and inter-examiner reproducibility of each ultrasound attenuation examination platform [23,31-33]. In previous studies, all three platforms (ATI, TAl, and UGAP) showed high intra-examiner and inter-examiner reproducibility (ICCS for intra-examiner and inter-examiner reproducibility: 0.93 and 0.79 for ATI; 0.99 and 0.98 for TAl; and 0.86 and 0.64 for UGAP, respectively) [23,31-33]. Considering the high inter-session reproducibility of each platform, each ultrasound attenuation examination technique can be used as a screening test or as a tool for monitoring treatment in patients with hepatic steatosis.

The present study had several limitations. First, this was a single-center study comprising a small number of patients; further multicenter and multi-platform studies are needed. Second, a histological diagnosis or MRI-PDFF as a noninvasive reference standard for hepatic steatosis was not performed in this study, as its primary goal was to evaluate the inter-platform reproducibility of ultrasound attenuation examination platforms and not to compare the diagnostic performance of each platform. Third, as one radiologist performed two sessions with each ultrasound system for each patient, inter-examiner reproducibility could not be assessed.

In conclusion, the results of this study indicate significant inter-platform variability across different ultrasound attenuation examination platforms, although the inter-session reproducibility of $A C$ values of each platform was excellent. Therefore, $A C$ values measured using different ultrasound attenuation examination techniques should not be used interchangeably for the longitudinal follow-up of patients with NAFLD.

ORCID: Sun Kyung Jeon: https://orcid.org/0000-0002-8991-3986; Jeong Min Lee: https://orcid.org/0000-0003-0561-8777; ljin Joo: https://orcid.org/0000-0002-13414072; Jeong Hee Yoon: https://orcid.org/0000-0002-9925-9973

\section{Author Contributions}

Conceptualization: Jeon SK, Lee JM. Data acquisition: Jeon SK, Lee JM. Data analysis or interpretation: Jeon SK, Joo I, Yoon JH. Drafting of the manuscript: Jeon SK. Critical revision of the manuscript: Lee $J M, J 00$ I, Yoon JH. Approval of the final version of the manuscript: all authors.

\section{Conflict of Interest}

Jeong Min Lee received grants from Samsung Medison, grants from Philips Healthcare, grants from GE Healthcare, grants from Canon Medical, personal fees and non-financial support from Siemens Healthcare, grants from RF MEDICAL, grants and personal fees from Bayer Healthcare, and grants and personal fees from Guerbet, outside the submitted work. Sun Kyung Jeon, ljin Joo, and Jeong Hee Yoon have declared no conflicts of interest.

\section{Acknowledgments}

This study was supported by Research Fund of the Korean Society of Ultrasound in Medicine for 2021.

\section{Supplementary Material}

Supplementary Table 1. Inter-platform reproducibility of AC values between three ultrasound attenuation examination platforms according to visual grade of hepatic steatosis (https://doi. org/10.14366/usg.21167).

Supplementary Table 2. AC values obtained using different ultrasound attenuation examination platforms according to number of acquisitions (https://doi.org/10.14366/usg.21167).

\section{References}

1. Loomba R, Sanyal AJ. The global NAFLD epidemic. Nat Rev Gastroenterol Hepatol 2013;10:686-690.

2. Vernon G, Baranova A, Younossi ZM. Systematic review: the epidemiology and natural history of non-alcoholic fatty liver disease and non-alcoholic steatohepatitis in adults. Aliment Pharmacol Ther 2011;34:274-285. 
3. Pais R, Barritt AS 4th, Calmus $Y$, Scatton O, Runge $T$, Lebray $P$, et al. NAFLD and liver transplantation: current burden and expected challenges. J Hepatol 2016;65:1245-1257.

4. Friedman SL, Neuschwander-Tetri BA, Rinella M, Sanyal AJ. Mechanisms of NAFLD development and therapeutic strategies. Nat Med 2018;24:908-922.

5. Machado MV, Cortez-Pinto $H$. Non-invasive diagnosis of nonalcoholic fatty liver disease: a critical appraisal. J Hepatol 2013;58:1007-1019.

6. Tang A, Desai A, Hamilton G, Wolfson T, Gamst A, Lam J, et al. Accuracy of MR imaging-estimated proton density fat fraction for classification of dichotomized histologic steatosis grades in nonalcoholic fatty liver disease. Radiology 2015;274:416-425.

7. Yokoo T, Serai SD, Pirasteh A, Bashir MR, Hamilton G, Hernando $D$, et al. Linearity, bias, and precision of hepatic proton density fat fraction measurements by using MR imaging: a meta-analysis. Radiology 2018;286:486-498.

8. Hernaez R, Lazo M, Bonekamp S, Kamel I, Brancati FL, Guallar E, et al. Diagnostic accuracy and reliability of ultrasonography for the detection of fatty liver: a meta-analysis. Hepatology 2011;54:10821090.

9. Zhang E, Wartelle-Bladou C, Lepanto L, Lachaine J, Cloutier G, Tang A. Cost-utility analysis of nonalcoholic steatohepatitis screening. Eur Radiol 2015;25:3282-3294.

10. Dasarathy S, Dasarathy J, Khiyami A, Joseph R, Lopez R, McCullough AJ. Validity of real time ultrasound in the diagnosis of hepatic steatosis: a prospective study. J Hepatol 2009;51:1061-1067.

11. Zhou Z, Zhang Q, Wu W, Lin YH, Tai DI, Tseng JH, et al. Hepatic steatosis assessment using ultrasound homodyned-K parametric imaging: the effects of estimators. Quant Imaging Med Surg 2019;9:1932-1947.

12. Lin SC, Heba E, Wolfson T, Ang B, Gamst A, Han A, et al. Noninvasive Diagnosis of nonalcoholic fatty liver disease and quantification of liver fat using a new quantitative ultrasound technique. Clin Gastroenterol Hepatol 2015;13:1337-1345.

13. Yang KC, Liao YY, Tsui PH, Yeh CK. Ultrasound imaging in nonalcoholic liver disease: current applications and future developments. Quant Imaging Med Surg 2019;9:546-551.

14. Paige JS, Bernstein GS, Heba E, Costa EA, Fereirra M, Wolfson $T$, et al. A pilot comparative study of quantitative ultrasound, conventional ultrasound, and MRI for predicting histologydetermined steatosis grade in adult nonalcoholic fatty liver disease. AJR Am J Roentgenol 2017;208:W168-W177.

15. Ferraioli G, Soares Monteiro LB. Ultrasound-based techniques for the diagnosis of liver steatosis. World J Gastroenterol 2019;25:6053-6062.

16. Taylor KJ, Riely CA, Hammers L, Flax S, Weltin G, Garcia-Tsao G, et al. Quantitative US attenuation in normal liver and in patients with diffuse liver disease: importance of fat. Radiology 1986;160:65-71.
17. Eddowes PJ, Sasso M, Allison M, Tsochatzis E, Anstee QM, Sheridan $D$, et al. Accuracy of FibroScan controlled attenuation parameter and liver stiffness measurement in assessing steatosis and fibrosis in patients with nonalcoholic fatty liver disease. Gastroenterology 2019;156:1717-1730.

18. Lv S, Jiang S, Liu S, Dong Q, Xin Y, Xuan S. Noninvasive quantitative detection methods of liver fat content in nonalcoholic fatty liver disease. J Clin Transl Hepatol 2018;6:217-221.

19. Pirmoazen AM, Khurana A, El Kaffas A, Kamaya A. Quantitative ultrasound approaches for diagnosis and monitoring hepatic steatosis in nonalcoholic fatty liver disease. Theranostics 2020; 10:4277-4289.

20. Bae JS, Lee DH, Lee JY, Kim H, Yu SJ, Lee JH, et al. Assessment of hepatic steatosis by using attenuation imaging: a quantitative, easy-to-perform ultrasound technique. Eur Radiol 2019;29:64996507.

21. Jeon SK, Lee JM, Joo I, Park SJ. Quantitative ultrasound radiofrequency data analysis for the assessment of hepatic steatosis in nonalcoholic fatty liver disease using magnetic resonance imaging proton density fat fraction as the reference standard. Korean J Radiol 2021;22:1077-1086.

22. Tada T, Kumada T, Toyoda H, Kobayashi N, Sone Y, Oguri T, et al. Utility of attenuation coefficient measurement using an ultrasoundguided attenuation parameter for evaluation of hepatic steatosis: comparison with MRI-determined proton density fat fraction. AJR Am J Roentgenol 2019;212:332-341.

23. Jeon SK, Lee JM, Joo I. Clinical feasibility of quantitative ultrasound imaging for suspected hepatic steatosis: intra- and inter-examiner reliability and correlation with controlled attenuation parameter. Ultrasound Med Biol 2021;47:438-445.

24. Hamaguchi M, Kojima T, Itoh Y, Harano Y, Fujii K, Nakajima T, et al. The severity of ultrasonographic findings in nonalcoholic fatty liver disease reflects the metabolic syndrome and visceral fat accumulation. Am J Gastroenterol 2007;102:2708-2715.

25. Shrout PE, Fleiss JL. Intraclass correlations: uses in assessing rater reliability. Psychol Bull 1979;86:420-428.

26. Koo TK, Li MY. A guideline of selecting and reporting intraclass correlation coefficients for reliability research. J Chiropr Med 2016;15:155-163.

27. Kobus T, van der Laak JA, Maas MC, Hambrock T, Bruggink CC, Hulsbergen-van de Kaa CA, et al. Contribution of histopathologic tissue composition to quantitative MR spectroscopy and diffusionweighted imaging of the prostate. Radiology 2016;278:801-811.

28. Han A, Zhang YN, Boehringer AS, Andre MP, Erdman JW Jr, Loomba $R$, et al. Inter-platform reproducibility of ultrasonic attenuation and backscatter coefficients in assessing NAFLD. Eur Radiol 2019;29:4699-4708.

29. Han A, Andre MP, Deiranieh L, Housman E, Erdman JW Jr, Loomba R, et al. Repeatability and reproducibility of the ultrasonic attenuation 
coefficient and backscatter coefficient measured in the right lobe of the liver in adults with known or suspected nonalcoholic fatty liver disease. J Ultrasound Med 2018;37:1913-1927.

30. Ferraioli G, Maiocchi L, Savietto G, Tinelli C, Nichetti M, Rondanelli $M$, et al. Performance of the attenuation imaging technology in the detection of liver steatosis. J Ultrasound Med 2021;40:1325-1332.

31. Jeon SK, Lee JM, Joo I, Yoon JH, Lee DH, Lee JY, et al. Prospective evaluation of hepatic steatosis using ultrasound attenuation imaging in patients with chronic liver disease with magnetic resonance imaging proton density fat fraction as the reference standard. Ultrasound Med Biol 2019;45:1407-1416.

32. Fujiwara Y, Kuroda H, Abe T, Ishida K, Oguri T, Noguchi S, et al. The B-mode image-guided ultrasound attenuation parameter accurately detects hepatic steatosis in chronic liver disease. Ultrasound Med Biol 2018;44:2223-2232.

33. Yoo J, Lee JM, Joo I, Lee DH, Yoon JH, Kang HJ, et al. Reproducibility of ultrasound attenuation imaging for the noninvasive evaluation of hepatic steatosis. Ultrasonography 2020;39:121-129. 